\title{
El modelo de promoción de la salud de Nola Pender. \\ Una reflexión en torno a su comprensión
}

\section{The model of health promotion proposed by Nola Pender. A reflection on your understanding}

Lic. Gladis Patricia Aristizábal Hoyos•

Lic. Dolly MarleneBlanco Borjas•

Lic. Araceli Sánchez Ramos •

Mtra. Rosa María Ostiguín Meléndez ••

\section{Resumen}

La comprensión de las teorías y modelos de enfermería llevan consigo un amplio recorrido de discusión que contribuye al crecimiento de la disciplina. El Modelo de Promoción de la Salud propuesto por Nola Pender, es ampliamente utilizado por los profesionales de enfermería, ya que permite comprender comportamientos humanos relacionados con la salud, y a su vez, orienta hacia la generación de conductas saludables. El objetivo del presente trabajo fue realizar un análisis de los artículos originales publicados en torno a este modelo, durante el período 2000-2010, identificando los aspectos más relevantes expresados en dichas publicaciones, así como las formas en que éste es interpretado y utilizado en la práctica.

Las investigaciones realizadas a partir de este modelo, en el periodo mencionado, predominan en Brasil, Estados Unidos de América y México. Los grupos de personas en las que más se hacen, son adolescentes y adultos, las temáticas más mencionadas son: actividad física, estilos de vida saludable, enfermedades crónicas y obesidad, la gran mayoría de los investigadores, utiliza la totalidad de conceptos expresados en el modelo para la interpretación del fenómeno estudiado.

Los profesionales que más investigan en torno a este modelo, son de los campos de enfermería y sociología. Se ha notado un incremento de las investigaciones alrededor del Modelo de Promoción de la Salud en la última década, reflejo del interés de estos expertos por comprender las conductas promotoras de la salud, así como de las motivaciones que llevan a realizarlas.

-Alumnas de la Maestría en Enfermería 9a generación, ENEO-UNAM.

..Asesora, coordinadora del programa de Maestría en Enfermería ENEO - UNAM.

correspondencia: aristizabalh.patricia@gmail.com

RECIBIDO: 30 MAYO 2011

ENVIADO: 6 JUNIO 2011

ACEPTADO: 12 SEPTIEMBRE 2011 


Key
Words:
Models nursing,
Promotion of health;
Nola Pender;
Nursing research

Abstract

Comprehension of theories and Nursing Models comprise a wide range of discussions contributing to the growth of discipline. The model of health promotion proposed by Nola Pender is widely used by Nursing professionals; as it leads to the understanding of human behavior related to health; it also leads to the development of healthy behavior. The aim of this work is to perform an analysis of the original articles published regarding this model, carried out between 2000 and 2010 in America identifying the most relevant issues stated under such publications, and the ways it is interpreted in real practice.

Research performed from this model in the above stated period of time, is mostly used in Brazil, the United States and Mexico. The groups of people widely involved are youngsters, adults and elders, most used topics are: physical activity, healthy life styles, chronicle diseases and the application of the model instruments, most of the researches use the whole of the concepts expressed in the model for the interpretation of the surveyed phenomenon.

Nurses and Sociologists are the professionals performing most of the research of this model. An increase of HMP (Health Model Promotion) research has been detected in the last decade, evidencing the interest of these experts to understand the Promotional Health Behaviors, as well as the motivational aspects that lead them to perform them.

\section{INTRODUCCIÓN}

Nola Pender, enfermera, autora del Modelo de Promoción de la Salud (MPS), expresó que la conducta está motivada por el deseo de alcanzar el bienestar y el potencial humano. Se interesó en la creación de un modelo enfermero que diera respuestas a la forma cómo las personas adoptan decisiones acerca del cuidado de su propia salud.

El MPS pretende ilustrar la naturaleza multifacética de las personas en su interacción con el entorno cuando intentan alcanzar el estado deseado de salud; enfatiza el nexo entre características personales y experiencias, conocimientos, creencias y aspectos situacionales vinculados con los comportamientos o conductas de salud que se pretenden lograr.

Nola Pender nace en Lansing, Michigan el 16 de Agosto de 1941, su primer acercamiento con la enfermería fue a los 7 años, al observar el cuidado enfermero que le ofrecían a su tía hospitalizada. En 1962 recibe su diploma de la escuela de enfermería de West Suburban Hospital de Oak Park, Illinois. En1964 completa su título en la Universidad del Estado de Michigan, Evanston, Illinois. Su trabajo de doctorado fue encaminado a los cambios evolutivos advertidos en los procesos de codificación de la memoria inmediata de los niños, de ahí surgió un fuerte interés por ampliar su aprendizaje en el campo de la optimización de la salud humana, que daría origen posteriormente al Modelo de Promoción de la Salud (MPS). ${ }^{1}$

El MPS expone de forma amplia los aspectos relevantes que intervienen en la modificación de la conducta de los seres humanos, sus actitudes y motivaciones hacia el accionar que promoverá la salud. Está inspirado en dos sustentos teóricos: la teoría de aprendizaje social de Albert Bandura ${ }^{2,3}$ y el modelo de valoración de expectativas de la motivación humana de Feather. ${ }^{4}$

El primero, postula la importancia de los procesos cognitivos en el cambio de conducta e incorpora aspectos del aprendizaje cognitivo y conductual, reconoce que los factores psicológicos influyen en los comportamientos de las personas. Señala cuatro requisitos para que éstas aprendan y modelen su comportamiento: atención (estar expectante ante lo que sucede), retención (recordar lo que uno ha observado), reproducción (habilidad de reproducir la conducta) y 
motivación (una buena razón para querer adoptar esa conducta).

El segundo sustento teórico, afirma que la conducta es racional, considera que el componente motivacional clave para conseguir un logro es la intencionalidad. De acuerdo con esto, cuando hay una intención clara, concreta y definida por conseguir una meta, aumenta la probabilidad de lograr el objetivo. La intencionalidad, entendida como el compromiso personal con la acción, constituye un componente motivacional decisivo, que se representa en el análisis de los comportamientos voluntarios dirigidos al logro de metas planeadas.
El MPS expone cómo las características y experiencias individuales así como los conocimientos y afectos específicos de la conducta llevan al individuo a participar o no en comportamientos de salud, toda esta perspectiva Pender la integra en el siguiente diagrama del Modelo de Promoción de la Salud: (Véase gráfico inferior).

En este esquema se pueden ubicar los componentes por columnas de izquierda a derecha; la primera trata sobre las características y experiencias individuales de las personas y abarca dos conceptos: conducta previa relacionada y los factores personales.
El primer concepto se refiere a experiencias anteriores que pudieran tener efectos directos e indirectos en la probabilidad de comprometerse con las conductas de promoción de la salud. El segundo concepto describe los factores personales, categorizados como biológicos, psicológicos y socioculturales, los cuales de acuerdo con este enfoque son predictivos de una cierta conducta, y están marcados por la naturaleza de la consideración de la meta de las conductas.

Los componentes de la segunda columna son los centrales del modelo y se relacionan con los conocimientos y afectos (sentimien-

\section{Características y Experiencias individuales \\ Cogniciones $y$ afectos relativos a la conducta especifica}

\section{Resultado conductual}

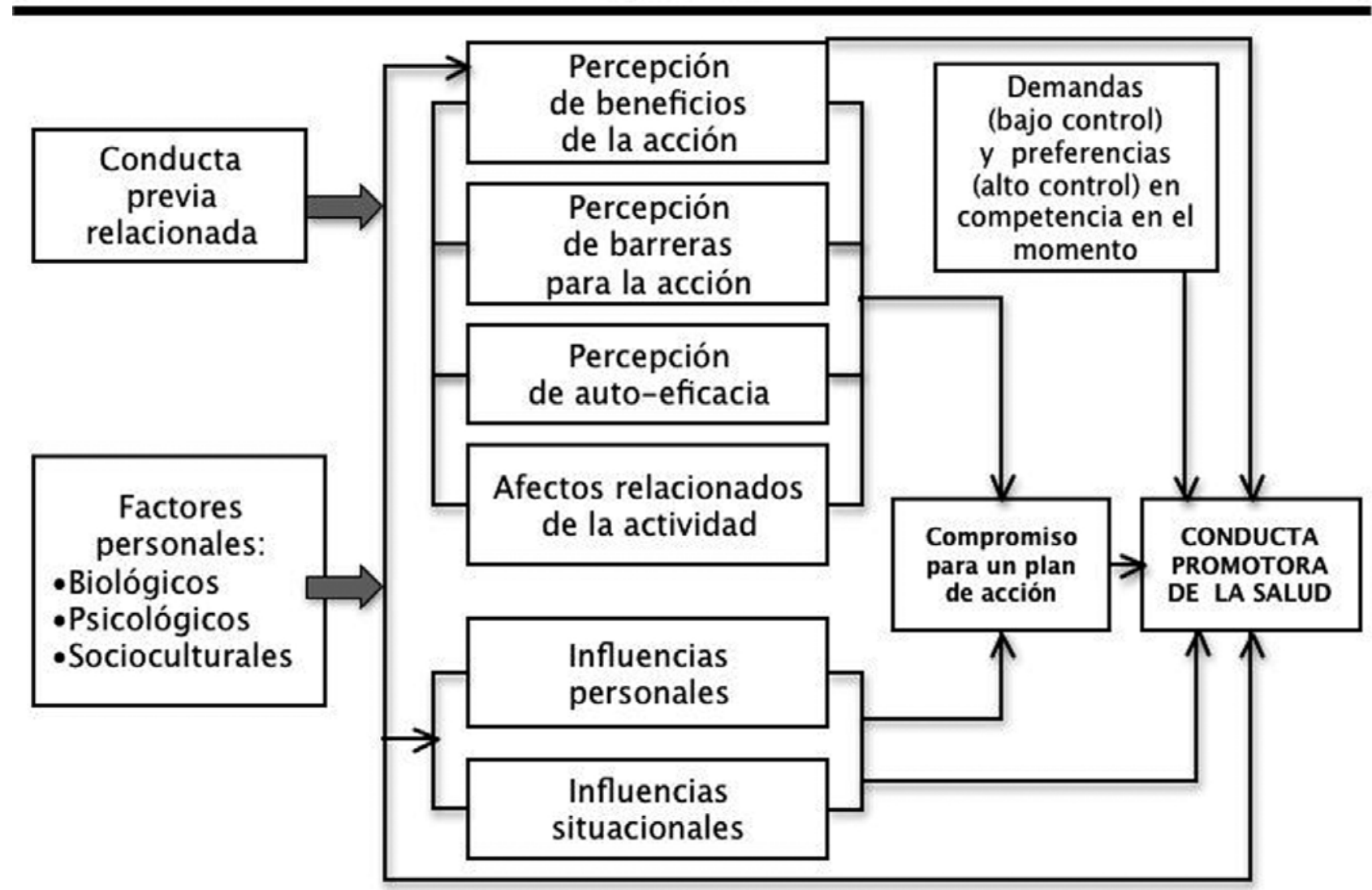

Fuente: Modelo de promoción de la Salud de Pender1996. En: Cid PH, Merino JE, Stiepovich JB ${ }^{21}$ 
tos, emociones, creencias) específicos de la conducta, comprende 6 conceptos; el primero corresponde a los beneficios percibidos por la acción, que son los resultados positivos anticipados que se producirán como expresión de la conducta de salud; el segundo se refiere a las barreras percibidas para la acción, alude a las apreciaciones negativas o desventajas de la propia persona que pueden obstaculizar un compromiso con la acción, la mediación de la conducta y la conducta real; el tercer concepto es la auto eficacia percibida, éste constituye uno de los conceptos más importantes en este modelo porque representa la percepción de competencia de uno mismo para ejecutar una cierta conducta, conforme es mayor aumenta la probabilidad de un compromiso de acción y la actuación real de la conducta. La eficacia percibida de uno mismo tiene como resultado menos barreras percibidas para una conducta de salud específica. El cuarto componente es el afecto relacionado con el comportamiento, son las emociones o reacciones directamente afines con los pensamientos positivos o negativos, favorables o desfavorables hacia una conducta; el quinto concepto habla de las influencias interpersonales, se considera más probable que las personas se comprometan a adoptar conductas de promoción de salud cuando los individuos importantes para ellos esperan que se den estos cambios e incluso ofrecen ayuda o apoyo para permitirla; finalmente, el último concepto de esta columna, indica las influencias situacionales en el entorno, las cuales pueden aumentar o disminuir el compro- miso o la participación en la conducta promotora de salud.

Los diversos componentes enunciados se relacionan e influyen en la adopción de un compromiso para un plan de acción, concepto ubicado en la tercera columna y que constituye el precedente para el resultado final deseado, es decir para la conducta promotora de la salud; en este compromiso pueden influir además, las demandas y preferencias contrapuestas inmediatas. En las primeras se consideran aquellas conductas alternativas sobre las que los individuos tienen un bajo control porque existen contingentes del entorno, como el trabajo o las responsabilidades del cuidado de la familia, en cambio las preferencias personales posibilitan un control relativamente alto sobre las acciones dirigidas a elegir algo. En síntesis, el MPS plantea las dimensiones y relaciones que participan para generar o modificar la conducta promotora de la salud, ésta es el punto en la mira o la expresión de la acción dirigida a los resultados de la salud positivos, como el bienestar óptimo, el cumplimiento personal y la vida productiva. ${ }^{1}$

Vista así, la promoción de la salud es una actividad clave en los diferentes ámbitos en que participan los profesionales de enfermería, esta consideración fundamenta la relevancia de analizar los trabajos que se han realizado en torno a dicho modelo a fin de identificar los intereses que orientan su uso y los hallazgos de las investigaciones. Para la búsqueda de artículos se definió el periodo del año 2000 al 2010 en las bases de datos LILACS, SCIELO, REDALYC Y MEDLINE, publicados en idioma portugués, inglés y/o español; se utilizaron los

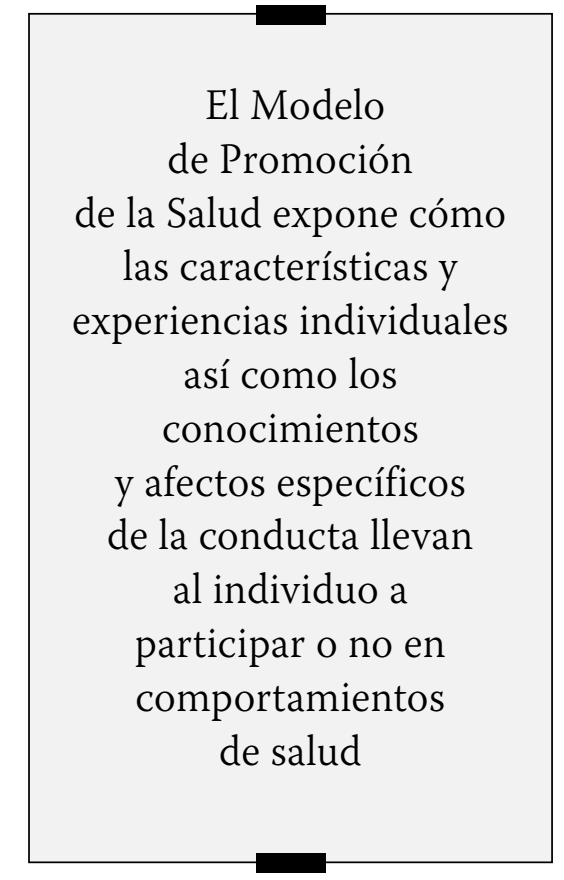

siguientes descriptores: modelo de promoción de la salud, Nola Pender, enfermería e investigación.

Se identificaron 38 documentos, de los cuales 25 corresponden a artículos en que explícitamente señalan que se utilizaron componentes del modelo para evaluar o promover alguna conducta de salud. ${ }^{5-29}$ Los otros 13 comprenden trabajos de revisión y ensayos que resaltan la importancia del modelo o de manera general entre sus fundamentos teóricos consideran éste. ${ }^{30-42}$ También en este segundo grupo se identificaron estudios enfocados a probar instrumentos para abordar algunos de los componentes del modelo.

El análisis que se realiza en el presente trabajo se centró en el primer subconjunto de estas publicaciones (artículos de carácter científico), con el fin de identificar los aspectos más relevantes del Modelo de Promoción de la Salud, así como la manera en que es interpretado y utilizado en la práctica.

Durante el periodo mencionado, se identificó un número signi- 
ficativo de publicaciones en las que se menciona el MPS, se observó que los países donde más se han realizado investigaciones con el modelo de Pender son: Brasil, Estados Unidos y México. Es importante señalar que en el mismo periodo se identificaron 6 estudios de investigación en los que participó la autora para probar su modelo en diferentes poblaciones ${ }^{5-10}$ también que los adolescentes y adultos son los grupos predominantes en las investigaciones identificadas. ${ }^{5-21}$

Los profesionales que más investigan acerca del modelo, son los profesionales de enfermería con formación en maestría $a^{9,11,13,15-17,21,23,28,29}$ y doctora$\mathrm{do}^{8,16,17,21,23,28,29}$ algunos apoyados de sociólogos ${ }^{21,36,42 .}$ Lo cual es una muestra de la simbiosis con profesiones como la sociología para entender los comportamientos de las personas y comunidades y saber como actuar en consecuencia.

Se encontraron investigaciones bajo una perspectiva metodológica cualitativa 5, 6, 17,22 así como cuantitativa ${ }^{7,8,9,10-21,23-25,29}$ Las primeras guardan prioritariamente relación con intervenciones en adolescentes y las segundas tienen una temática diversa. El tipo de estudios muestra la preferencia de las(os) enfermeras(os) por elegir metodologías cuantitativas para abordar los fenómenos estudiados y la aun escasa exploración de las cualitativas para la comprensión de los mismos.

Los diseños de estudios más utilizados fueron los descriptivos, transversales ${ }^{11,12,16,18-20,22}$ así mismo, se encontró un estudio socio poético desarrollado en Brasil ${ }^{22}$ que refleja la diversidad y versatilidad del modelo y la creatividad de los pro-

fesionales para abordar fenómenos de su interés.

Las temáticas más utilizadas en los artículos explorados fueron: actividad física y ejercicio, $5,7,11,24,25$ tanto en adolescentes como en adultos, estilos de vida saludable. ${ }^{19,21}$ Lo cual nos hace suponer que éstas son acciones eficientes en la modificación de la conducta y tienen impacto sobre las personas y su salud.

Por otro lado, las investigaciones en adolescentes y escolares muestran que si bien estos grupos tienen conductas aprendidas que no son fáciles de modificar, también cuentan con motivaciones diferentes y elementos capaces de influir en sus estilos de vida, de forma favorable.

La exploración del MPS puede ayudar a profesionales de la salud a entender el proceso salud-enfermedad, así como a apoyar la práctica de enfermería. En las investigaciones se explora el diagrama propuesto en el modelo, con el fin de comprender su utilidad en el cuidado de las personas. Este análisis ha permitido evidenciar que la representación gráfica del diagrama es de fácil comprensión, los componentes se describen en los textos con claridad lo que al parecer facilita su uso por

Todos los conceptos incluidos en el diagrama son mencionados en los estudios como una base importante para su desarrollo, sin embargo el más utilizado es el de autoeficacia las enfermeras en el cuidado e investigación, así el MPS puede ser una propuesta para llevar a cabo intervenciones de enfermería en el contexto de promoción de la salud relacionadas con el cambio de comportamiento.

Vale la pena destacar que todos los conceptos incluidos en el diagrama son mencionados en los estudios como una base importante para su desarrollo, sin embargo el más utilizado es el de autoeficacia, ${ }^{6,7,19-21}$ a la cual se le atribuye gran parte de los resultados exitosos, dicha expresión, es definida como la capacidad de una persona de generar una conducta de salud, lo que explica su importancia en la evaluación de las investigaciones y la pauta que puede dar a la hora de intervenir en las necesidades de los individuos.

Derivado del análisis realizado se aprecia una gama de alternativas en la exploración del modelo, algunas investigaciones tienen preferencia para usarlo como explicación o comprensión de ciertas conductas de salud, como son: la actitud consciente hacia el uso de guantes, ${ }^{14}$ el uso de estrategias para realizar ejercicio, ${ }^{6,8,25}$ y la comprensión de fenómenos encaminados al bienestar. ${ }^{7,21}$

En adultos es ampliamente empleado con el propósito de dar respuestas a las conductas generadoras de salud que motivan a las personas a adquirir dichos comportamientos., ${ }^{9,15,16,19,22}$

Al cotejar la similitud entre el título, los objetivos, los resultados y las conclusiones de los artículos analizados, se puede observar que con relativa facilidad se asume que el modelo se aplica, aunque en sentido estricto más bien lo utilizan 
para la comprensión y explicación de realidades humanas, lo cual, es función de las teorías (de las cuales se derivan los modelos); ${ }^{43}$ es decir, se utilizan los modelos como base teórica. ${ }^{44}$

En este orden de ideas, algunas interrogantes surgidas de este análisis son en primer lugar en el sentido de la interpretación del modelo por los profesionales de enfermería, pues parece asumirse la validez y sentido de las relaciones entre los componentes sin una aparente discusión a profundidad de estos aspectos.

También de acuerdo con el modelo, los beneficios o barreras para la acción aparentemente están modulados por las características y experiencias personales, sin embargo nos parece importante documentar de manera más contundente ante qué características o experiencias tienen mayor peso los beneficios o bien ante cuáles las barreras. $\mathrm{O}$ bien, ¿qué tiene mayor influencia en generar conductas relacionadas con la salud, los beneficios y barreras percibidos ó las demandas por cubrir las necesidades básicas y preferencias? ¿cuál es la correspondencia existente entre lo que plantea el modelo con la realidad observada?

\section{Conclusiones}

El Modelo de Promoción de la Salud ha sido utilizado por los profesionales de Enfermería en la última década, con frecuencia en diferentes situaciones que van desde la práctica segura del uso de guantes en las enfermeras hasta la percepción de la salud de los pacientes, pero particularmente dirigido a la promoción de conductas saludables en las personas, lo que indudablemente es una parte esencial del cuidado enfermero tal como se evidencia en los trabajos publicados los cuáles conservan un interés genuino e implícito hacia el cuidado, el mejoramiento de la salud y la generación de conductas que previenen la enfermedad.

Este modelo parece ser una poderosa herramienta utilizada por las(os) enfermeras(os) para comprender y promover las actitudes, motivaciones y acciones de las personas particularmente a partir del concepto de autoeficacia, señalado por Nola Pender en su modelo, el cual es utilizado por los profesionales de enfermería para valorar la pertinencia de las intervenciones y las exploraciones realizadas en torno al mismo.

Sería deseable documentar en mayor medida el sentido de las relaciones planteadas en el modelo a fin de fortalecer su solidez teórica y empírica, por lo que seguramente en un futuro surgirán nuevas interrogantes alrededor de esta propuesta.

Podemos concluir que conocer y explorar el tipo de investigaciones que se realizan, la relación de éstas con las teorías y constructos propios de la disciplina, la utilidad de las mismas y su impacto en la consecución de mejores oportunidades y calidad de vida, fortalece nuestra disciplina y fundamenta su quehacer ya que orienta de manera objetiva hacia los intereses de los profesionales y las necesidades de los pacientes.

\section{REFERENCIAS BIBLIOGRÁFICAS}

1. Sakraida J. Modelo de Promoción de la Salud. Nola J Pender. En: Maerriner TA, Rayle-Alligood M, editores. Modelos y teorías de enfermería, $6^{\circ}$ ed. España: Elsevier-
Mosby; 2007.

2. Worchel S. Cooper J, Goethals GR. Olson J. Psicología Social. Madrid: Thomson Editores 2002.

3. Bandura A. Self-efficacy: Toward a unifyng theory of behavioral change. Psychology review, 1977; 84(2): 191-215.

4. Feather NT. Espectations and actions: Expectancy-value models in psychology. Hillsdale, NJ: Lawrence Erlbaum Associates, Inc. 1982.

5. Garcia WA, Norton BMA, Fern M, Coviak C, Pender N, Ronis LD. Gender and Developmental Differences in Exercise Beliefs Among Youth and Prediction of Their Exercise Behavior. Journal of School Health [internet] 1995 [consultado el 26 de marzo del 2011]; 65(6):[213-219]. Disponible en: http://bit.ly/ACcJ0l

6. Robbins LB, Pis MB, Pender NJ, Kazanis AS. Physical activity selfdefinition among adolescents. Res Theory Nurs Pract [internet] 2004 [consultado el 25 de marzo del 2011]; 18(4): [317-30]. Disponible en: http://1.usa.gov/yhqUeM

7. Robbins LB, Pender NJ, Ronis DL, Kazanis AS, Pis MB. Physical activity, self-efficacy, and perceived exertion among adolescents. Res Nurs Health [internet] 2004 [consultado el 25 de marzo del 2011]; 27(6): [435-46]. Disponible en: http://1.usa.gov/yu7vOz

8. Wu TY, Pender NJ, Yang KP. Promoting physical activity among Taiwanese and American adolescents. J Nurs Res [internet] 2002 [consultado el 24 de marzo del 2011];10(1):[57-64]. Disponible en: http://1.usa.gov/wM3mRQ

9. Shin Y, Yun S, Pender N, Jang H. Test of the health promotion model as a causal model of commitment to a plan for exercise among Korean 
adults with chronic disease. Research in Nursing \& Health [internet] 2005 [consultado el 27 de febrero del 2011]; 28(2):[117-125]. Disponible en: http://bit.ly/ybZfVf 10. Pender NJ, Pender AR. Illness prevention and health promotion services provided by nurse practitioners: predicting potential consumers. Am J Public Health [internet] 1980 [consultado el 26 de marzo del 2011]; 70(8):[798-803]. Disponible en: http://1.usa.gov/ xPja5I

11. Durán MR. Relación entre los factores cognitivos perceptuales y modificantes, con las conductas promotoras de salud. Tesis Universidad de Venezuela [Internet] Caracas; 1998. [Consultado el 25 de marzo del 2011] Disponible en: http://bit.ly/yMoZIv

12. Jeremy KA, Roy AS, Smyth P. Coronary Artery Disease and Smoking Cessation Intervention by Primary Care Providers in a Rural Clinic. Online Journal of Rural Nursing and Health Care [internet]. 2009 [consultado el 27 de marzo del 2011]; 9(2):[82-94]. Disponible en: http://bit.ly/AnuebJ

13. Trejo MF. Aplicación del modelo de Nola Pender a un adolescente con sedentarismo. Enf.Neurológica, 2010. 9(1):39-44.

14. MarzialeI HM, Dos Santos AZ, FelliI EV, Hideko MA. Rede de Prevenção de Acidentes de Trabalho: uma estratégia de ensino a distancia. Rev enferm. [Internet] 2010 [consultado el 25 de marzo del 2011]; 63(2): [250-256]. Disponible en: http://bit.ly/wSKBFa

15. Dos Santos I, Dos Santos CS, Miguéis LB. Promovendo autocuidado em clientes com coronariopatia: aplicação do diagrama de Nola Pender. Revista de Pesquisa Cuida- do é Fundamental Online Qualis [internet]. 2010 América do Norte [consultado el 26 de marzo del 2011];2(Ed.Supl):[266-270].Disponible en: http://bit.ly/zlBPvQ

16. Gallegos EC, Bañuelos Y. Conductas protectoras de salud en adultos con diabetes mellitus tipo II. Investigación y educación en enfermería [internet] 2004[consultado el $26 \mathrm{de}$ marzo del 2011] 22(2). Disponible en: http://bit.ly/A4svXs

17. Dos Santos AZ, Palucci MM, Cruz MR. Práctica segura del uso de guantes en la punción venosa por los trabajadores de enfermería. Cienc. Enferm [internet]. 2006 [consultado el 26 de marzo del 2011]; 12(2):[63-72]. Disponible en: http://bit.ly/zAyrlx

18. Medina ML, Díaz AP, Barrientos EG, Peña DM. Percepción de salud y su efecto en pacientes con diabetes. av.enferm [internet] 2009 [consultado el 27 de marzo del 2011];27(2):[13-18]. Disponible en: http://bit.ly/z5UKQd

19. Triviño Z, Stiepovich J, Merino MJ. Factores predictores de conductas promotoras de salud en mujeres peri post-menopáusicas de Cali, Colombia. Colomb Med [Internet] 2007 [consultado el 25 de marzo de 2011]; 38: [395-407]. Disponible en: http://bit.ly/wD6CXa

20. Becerra MM, Díaz LH. Niveles de actividad física, beneficios, barreras y autoeficacia en un grupo de empleados oficiales. av.enferm. [Internet]. 2008 [consultado el 28 marzo 2011]; 26(2): 43-50. Disponible en: http://bit.ly/AnZBnv

21. Cid PH; Merino JE, Stiepovich JB. Factores biológicos y psicosociales predictores del estilo de vida promotor de salud. Rev Méd Chile [internet]. 2006[consultado el 25 Marzo 2011]; 134(12):[1491-
1499] . Disponible en: http://bit. ly/yQukZd

22. Dos Santos AS, Dos Santos I. Promoção do autocuidado de idosos para o envelhecer saudável: aplicação da teoria de nola pender. Texto \& Contexto Enfermagem. [Internet]. 2010 [ consultado el 25 de marzo 2011]; 19(4):[745-753]. Disponible en: http://bit.ly/yPM$\mathrm{pDu}$

23. Monteiro FM, Oliveira JC, Vitor FA, Leite de Araujo T, Barbosa LX. Avaliação do estado nutricional de crianças com cardiopatia congênita sob a ótica de pender. rev. enferm. uerj, Rio de Janeiro,[Internet].2009 [Consultado el 25 de Marzo 2011] 17(4):[581-588]. Disponible en: http://bit.ly/ywTjyF

24. Gomez NG, Pessoa RM, Frota TC; Leite de Araujo T, Barbosa LX. Students' physical activity: an analysis according to Pender's health promotion model. Rev Esc Enferm. São Paulo 2009.43(4):744-80.

25. Gomes N; Pessoa R; CavalcanteIII T; Leite de Araujo T; Barbosa L. Atividade física de escolares: análise segundo o modelo teórico de promoção da saúde de Pender. Rev. Enferm São Paulo [internet] 2009 [consultado el 25 de marzo del 2011] 43 (9): [744-780]. Disponible en: http://bit.ly/A5LeWa

26. Curry M. Increasing awareness of type 2 diabetes in adolescents through theatre. A Thesis presented to the Faculty of California State University.[Internet].2009 [consultado el 26 de marzo del 2011]. Disponible en: http://bit.ly/xja4L1

27. Wood W, "African American women and hypertension: their resources of information, knowledge level, and health promoting behaviors". Masters Theses and Docto- 
ral Dissertations papers 157 [Internet]. 2008 [consultado el 25 de marzo del 2011]. Disponible en: http://bit.ly/z2HmcT

28. Wu T, Pender NJ. A Panel Study of Physical Activity in Taiwanese Youth: Testing the Revised HealthPromotion Model. Family \& Community Health [internet] 2005. [Consultado el 27 de marzo del 2011]; 28(2)[113-124]. Disponible en: http://bit.ly/yOQWBV

29. Wu T, Pender NJ. Determinants of physical activity among Taiwanese adolescents: An application of the health promotion model. Research in Nursing and Health USA [internet] 2002. [consultado el 23 de marzo del 2011], 25(1):[25-36]. Disponible en: http://bit.ly/y7iVDT

30. Hernández MF, Márquez RO, Alvarado BT, Cortés EA. Intervención educativa en el manejo del recién nacido prematuro. Rev Enferm IMSS [Internet] 2000. [Consultado 27 de Marzo 2011]; 8(1):[21-25]. Disponible en: http:// bit.ly/zwNoSl

31. Bitencourt GR, Santana RF, Silva CC, Moreira ES. Identificação do diagnóstico de enfermagem autocontrole ineficaz de saúde nas doenças crônicas: validação de instrumento. $10^{\circ}$ SINAEn [Internet] 2010. [consultado el 26 de marzo del 2011] Trabalho 177 1/3. Disponible en: http://bit.ly/ z86TkY

32. Costa SS, Devos EB, Tarouco BD; CestariI EM, Lerch VL .Health promotion for the elderly: gerontogeriatric nursing commitment. Acta Paul Enferm São Paulo [Internet] 2008. [Consultado el $25 \mathrm{de}$ Marzo 2011] 21(4): [649-653]. Disponible en: http://bit.ly/wzDyjv

33. Dos Santos AS, Dos Santos I, Mi- guéis LB. Body image of the elderly in the reflex of self-care for healthy aging: A social-poetic study. Journal of Nursing, North America [internet] 2010 [consultado el 26 de marzo del 2011]; 9(1). Disponible en http://bit.ly/wGzxix

34. Edén BK, Orleans T, Mulrow DC, Pender NJ, Teutsch MS. Clinician Counseling to Promote Physical Activity. Agency for Healthcare Research and Quality. [internet] 2002. [Consultado el 23 de marzo del 2011]. Disponible en: http://1. usa.gov/wOxW5T

35. Galicia NR, Nájera GG, Morales AN., Villegas $M^{a} V$. Promoción de Conductas Saludables en Personas con Diabetes Mellitus tipo 2. Desarrollo Cientif Enferm-Méx [Internet]. 2009 [consultado el 27 de marzo del 2011]; 17 (8):[335357]. Disponible en: http://bit.ly/ zMfveh

36. Fonseca JV, Venicios de Olivera ML, Barbosa LX. Análise do diagrama do modelo de promoção da saúde de Nola J. Pender. Acta Paul. Enferm [Internete] 2005. [Consultado el 27 de Marzo de 2011];18(3):[235-240].Disponible en: http://bit.ly/znnzZn

37. Fonseca JV, Barbosa LX, Almeida CP. Adaptação transcultural para o Brasil da Exercise Benefi ts/BarriersScale (EBBS) para aplicação em idosos: uma avaliação semántica. Cad. Saúde Pública, Rio de Janeiro [internet] 2008.[Consultado el 26 de marzo del 2011]; 24(12):[2852-2860]. Disponible en: http://bit.ly/yTblOK

38. Miyar LO. Impacto de un programa de promoción de la salud aplicado por enfermería a pacientes diabéticos tipo 2 en la comunidad. Rev Latino-am Enfermagem [Internet] 2003. [Consultado el 25 de marzo del 2011];11(6):[713-719].Disponible en: http://bit.ly/wPzRL7

39. Padula AC, Sullivan M. Long-Term Married Couples' Health Promotion Behaviors: Identifying Factors That Impact Decision-Making. J Gerontol Nurs [internet] 2006. [Consultado el 25 de marzo del 2011]; 10(32): [37-47]. Disponible en: http://bit.ly/zbmg8S

40. Palucci MM, De Jesus CL. Modelos explicativos e de intervenção na promoção da saúde do trabalhador. Acta Paul Enferm [internet] 2008. [Consultado el 23 de marzo del 2011];21(4):[654-659]. Disponible en: http://bit.ly/zbLCek

41. Sampaio SR, Silva A, Cardoso MJ, Barboza N, Santos M, Amantéa LM. Risco cardiovascular: plano de ação à luz do modelo de promoção da saúde de nola pender. $61^{\circ}$ congresso Brasileiro do enfermagen [internet] del 2009. [Consultado el 25 de marzo del 2011] Trabalho 3199 - 1/3. Disponible en: http://bit.ly/xSh2fD

42. Walker SN, Sechrist KR; Pender NJ. The Health-Promoting Lifestyle Profile: Development and Psychometric Characteristics. Nurs Res [Internet] 1987. [Consultado el 25 de marzo del 2011]36(2):[7681]. Disponible en: http://1.usa. gov/zAOBg8

43. Marquis-Bishop S, Hardin S. Proceso de desarrollo de la teoría. En: Marriner-Tomey A, Raile Alligood M. Modelos y Teorías en Enfermería. Madrid: Elsevier 2007. pp 35-49.

44. Riehl-Sisca J. Modelos conceptuales de enfermería. Barcelona: Ediciones Doyma 1992. 